Proceedings of the 2012 Winter Simulation Conference

C. Laroque, J. Himmelspach, R. Pasupathy, O. Rose, and A. M. Uhrmacher, eds.

\title{
A GENERIC MODEL TO ASSESS SUSTAINABILITY OF RESOURCE MANAGEMENT PLANS WITHIN CONCURRENT REGULATORY CONTEXTS
}

\author{
Jean-Pierre Müller \\ CIRAD-GREEN \\ Campus International de Baillarguet \\ 34398 Montpellier cedex 5, FRANCE
}

\author{
Sigrid Aubert \\ CIRAD-GREEN and ESSA \\ Ampandrianomby, BP 853-99 \\ Antananarivo, MADAGASCAR
}

\begin{abstract}
Management of the renewable natural resources in Madagascar is gradually being transferred to the local communities. However, these local communities are struggling to assess the consequences of the management plans they must develop and implement on sustainability, within concurrent regulatory contexts. From this Malagasy case, we derived, from a law anthropology perspective, a generic model called MIRANA. From a social perspective, MIRANA formalizes institutions as sets of constitutive and regulatory norms, defining multiple layered territories, and multiple perspectives on the agents and resources. From an individual perspective, MIRANA specifies agents' behaviors as a combination of subsistence, production, and contractual relations, accounting for a multiplicity of normative and incentive structures to compromise and to implement. MIRANA allows to analyze the impact on sustainability of agents' behaviors submitted to concurrent normative orders, in a context of law pluralism.
\end{abstract}

\section{INTRODUCTION}

Management of the renewable natural resources in Madagascar is gradually being transferred to the local communities (VOI for Vondron'Olona Ofotony in Malagasy). However, these VOIs are struggling to assess the consequences of the management plans they must develop and implement on sustainability. In (World Commission on Environment and Development 1987, p. 43): "Sustainable development is development that meets the needs of the present without compromising the ability of future generations to meet their own needs." The definition entails that the use of the resources provided by the natural environment should not exceed its renewal capacity (ecological sustainability), while maintaining the livelihood of the current and future generations (economical and social sustainability). The use of the resources depends both on the practices (the technological factors), and on the resource access regulations (the normative factors). Assuming the practices constant, we will focus on the normative factors. A number of resource access regulations are implemented by various territorial administrations: the natural park, the forest and communal administrations, and the customary communities. Our aim is to explore how the introduction of new norms (zoning, quotas, controls and sanctions) and economical tools (taxes, permits, incentives) by VOIs impacts their sustainability. The impact on the sustainability is evaluated at the ecological level by the evolution of the exploited specie populations, and at the economical and social level by the capacity to satisfy actors' needs and/or the number of illegal actions.

The MIRANA (Aubert, Müller, and Ralihalizara 2010) model has been developed to help the local communities to negotiate and set-up their management plans. To design MIRANA and following the steps advocated by the MIMOSA platform (Müller 2010), we developed a conceptual model, from a law anthropology perspective, that relies on a fundamental distinction between the studied system and its interpretations from various legal perspectives. The studied system is described with its actors, its resources (including space) and the processes and actions that are taken place therein. A legal perspective defines a terminology with roles played by subjects of law (physical or moral persons), objects of law 


\section{Müller, and Aubert}

(e.g., properties, deliverables) and activities (e.g., use, exploitation). The heterogeneity of the actors and the multiplicity of institutions lead to multiply the legal perspectives and their associated terminologies (Müller and Aubert 2011).

We propose to reify the legal perspectives by the notion of institution. Ostrom (2005) defines an institution as a terminology and a set of norms shared by a group of people from a given perspective. The group does not need to be aware of it. Searle (1995) distinguishes in an institution between the regulative norms and the constitutive norms. While the regulative norms describe the rights and duties associated to the various status or roles of the actors, the constitutive norms describe how aspects of the reality are counted as pertaining to given concepts. These concepts are used to express the regulative norms.

Multi-agent systems (MAS) have formalized a notion of institution. MOISE+ (Hübner, Sichman, and Boissier 2002) is focused on the distribution of tasks with three specifications of an institution: the structural specification defining the roles, the functional specification defining a hierarchy of goals and missions, and a deontic specification linking missions to roles. Esteva, Rosell, Rodriguez-Aguilar, and Arcos (2004) with AMELIA specifies the electronic institutions that impose protocols of interactions defined in deontic forms among agents. These approaches combine the organizational approach as AGR (Ferber and Gutknecht 1998) that defines the notion of groups of agents playing roles, and the normative approach insisting on the regulative norms (Boella and van der Torre 2004). The later as well as Grossi, Meyer, and Dignum (2008), Grossi (2007) explore the formalization of constitutive norms, but limited to reasoning about concepts. The use of constitutive norms to describe a concrete situation from a legal perspective is missing, hindering the possibility to account for multiple roles of the resources, lands and actors.

To represent resource management plans in a multiple regulatory context and to account for their impact on individual behaviors, we propose a two-level description. In the first level, we use the notion of institution as a set of constitutive and regulatory norms. A more complete formalization of constitutive norms using contextual ontologies, allows to naturally account for the notion of role and territory. In the second level, we use the notion of agent to represent both the individual actors on which the norms apply, and the collective actors to implement the regulatory norms of each institution. The norms are taken into account both at the individual level by constraining how the activities are planned and carried out, and at the collective level through various mechanisms of permits, control, sanctions and incentives.

We will first introduce our formalization of institutions and their constitutive and normative norms. Then, we will describe the agent structure, illustrated by concrete behavioral implementations.

\section{INSTITUTIONS AND NORMS}

We understand the notion of institution as a set of legal, practice or custom norms. This includes both constitutive and regulative norms. The norms can be taken directly into account by individual agents when producing their goal-directed behavior, and externally enforced by control and sanction or permit distribution mechanisms. This section will focus on the representation of the institutions and norms. The agents' architecture is described in the next section.

\subsection{Representation of Norms}

We use the distinction between constitutive and regulative norms as proposed in Searle (1995). Concerning the constitutive norms, we want to express, e.g., that:

1. "Eucalyptus counts as timber" understood as a relation between the concept of Eucalyptus from the forester's perspective and the concept of timber from the carpenter's perspective;

2. "This tree is (counts as) my property" that expresses a relationship defined between two individuals (here an object and an agent); 


\section{Müller, and Aubert}

3. 'Paul is (counts-as) a license holder' that associates an individual (Paul) to a concept (license holder). Similarly, "This area is (counts as) a protected zone" expresses an association between an individual (a geographic entity) and a concept (protected zone).

One recognizes the usual structure of the ontologies or description logics: the concepts (Eucalyptus, plant species, license holder, etc.), structured by taxonomic (plant specie is more general than Eucalyptus) and semantical (to be the property of) relationships, the individuals (this tree, Paul, this area) as instances of concepts (license holder), linked to one another (e.g., an area is included into another). But all these descriptions are contextual: the eucalyptus can be a plant specie only for the ecologist, Paul is a license holder or this area is a protected zone relative to a given institution. Finally, these relationships can be defined across contexts; the Eucalyptus from the ecologist's perspective is considered as fuel wood from the coal-man's perspective, this area from a surveyor's perspective is considered as a protected zone from the natural park administration's perspective. In these examples, there is no difference between putting an individual (Paul, this area) into a contextually defined category (license holder, protected zone) and attributing a role (the role of license holder, the role of protected zone) in this context. Grossi, Meyer, and Dignum (2008) provides a detailed analysis of the various meaning of "counting-as" in a context, namely the classificatory meaning, proper classification and being constitutive. Boella and van der Torre (2004) use it from the standpoint of a unique institution. However, in each of those cases, the analysis relies only on the concepts but not the individuals. Therefore it is not possible to account for the notion of role under the form of a contextual categorization as we propose.

The regulative norms are usually specified in logics using deontic operators (permission, obligation, prohibition). Differently, Boella and van der Torre (2004) formalizes the norms by violation criteria, the violation being inferred by constitutive norms. Indeed, the regulative norms raise the question of their control. In MAS software engineering, the norms are considered as high level specifications and are enforced directly in the design of the agents and their interactions. In this case, the deduction of a violation becomes a kind of program proof. Nevertheless, in open multi-agent systems, the case of agents that do not abide with the norms either intentionally or accidentally has to be taken into account (Dignum 2004). López y López, Luck, and d'Inverno (2002) proposes a mechanism of punishments and rewards, which requires the agent to reason on the advantages and disadvantages to obey or not to the norms.

We intends to use MIRANA to model the actual functioning of the institutional structures. In Law, for a norm to come into effect, one must foresee a control function that can be systematic or not and possibly leading to a violation record (the police function) and a sanction system in case of such a record (the penal function). In order to do that, we have separated the norm expression from its possible implementations. Thus a hunting quota can be enforced by a control strategy or by the distribution of licenses. Given the variety of implementations, we were brought to reify each institution by an agent having the status of a moral person and the role of manager of the associated institution. Therefore, we distinguish the institution as as structure, from the agent who manages it. We are now going to present our proposition to represent and implement the constitutive and regulative norms.

\subsection{The Formalization}

We formalize what precedes by using the contextual ontologies for the constitutive norms, and deontic forms for the regulative norms. Each institution $i \in I_{S M A}$ is defined as follows:

Definition 1 An institution $i$ is a pair $D I_{i}=\left\langle O_{i}, N_{i}\right\rangle$ where:

- $O_{i}$ is an ontology;

- $N_{i}$ is a set of regulative norms .

Having a family of institutions, we obtain a corresponding family of contextual ontologies. We will describe the ontologies and the regulative norms in turn. 


\subsubsection{Contextual Ontologies and Constitutive Norms}

For the constitutive norms, we define a family of ontologies $O_{i}$. Each ontology is defined on a language $\mathbb{L}_{i}=\left\langle\mathbb{C}_{i}, \mathbb{P}_{i}, \mathbb{O}_{i}, \mathbb{I}_{i}\right\rangle$ where:

- $\mathbb{C}_{i}$ is a set of concept names;

- $\mathbb{P}_{i}$ is a set of relation names (called roles in description logics);

- $\mathbb{O}_{i}$ is a set of individual (or object) names;

- $\mathbb{I}_{i}$ is a set of ontology names.

This definition is usual but the introduction of ontology names to internally refer to other ontologies. To account for the specificity of MAS, we decompose the set $\mathbb{C}_{i}$ of concepts into four disjoint sets:

- $\quad$ RRole $_{i}$ for the concepts categorizing the agents;

- $R_{R o l e}$ for the concepts categorizing the objects (or individuals);

- $A c t_{i}$ for the concepts categorizing the activities;

- $L o c_{i}$ for the concepts categorizing the places.

The derived concepts are built by the usual constructors: $\neg c, c_{1} \sqcup c_{2}, c_{1} \sqcap c_{2}, \forall r . c, \exists r . c, i: c$ where $c, c_{1}, c_{2}$ are the concepts, $r \in \mathbb{P}_{i}$ and $i \in \mathbb{I}_{i}$. $i: c$ denotes the concept $c$ in the ontology $i$ to denote the concepts defined in other ontologies. The set of derived concepts for the agents, objects, activities and places are disjoint.

Finally, $c_{1} \doteq c_{2}$ and $c_{1} \sqsubseteq c_{2}$ are the terminological axioms for definition and subsumption. Note that if $c_{1}, c_{2} \in L o c_{i}, c_{1} \sqcup c_{2}, c_{1} \sqcap c_{2}$ and $c_{1} \sqsubseteq c_{2}$ have the usual sense of geometrical intersection, union and inclusion. We can now formulate the first case:

- "Eucalyptus counts as timber" is expressed as Eucalyptus $\sqsubseteq j: T i m b e r$ where $j \in \mathbb{I}_{\text {forester }}$ is the name of the carpenter's ontology from the forester's perspective, or complementarily, i:Eucalyptus $\sqsubseteq$ Timber, $i$ being the name of the forester's ontology from the carpenter's perspective.

The forester can know that the eucalyptus is timber without the carpenter knowing it, or vice versa. Note that it is always necessary to mention in which ontology (from which point of view) the axiom is expressed because the denotation is strictly contextual. Thus, we obtain the expressivity of Grossi (2007). We just have added the locality of ontology names. Consequently, an ontology may not be able to designate another ontology and therefore may not know the corresponding concepts.

In the same way, we decompose the set $\mathbb{O}_{i}$ of individuals within:

- $A_{i}$ the set of agent names;

- $R_{i}$ the set of object names;

- $P_{i}$ the set of activity names;

- $L_{i}$ the set of place names.

The corresponding assertional axioms (or assertions) are $c(o)$ and $r\left(o_{1}, o_{2}\right)$ where $c \in \mathbb{C}_{i}, r \in \mathbb{P}_{i}$ or of the form $i: r$, and $o, o_{1}, o_{2} \in \mathbb{O}_{i}$ or of the form $i: o$, where $i \in \mathbb{I}_{i}$. $i: o$ denotes an individual $o$ in the ontology $i$ and allows denoting the individuals as named within another ontology. It is the same for the relations.

We can now express the last two examples:

- "This tree counts as my property" in $O_{\text {owner }}$ can be translated by $i$ :property $\left(\right.$ tree $\left._{27}, I\right)$ where $i$ is the name of the ontology of the institution in which the notion of property is defined, tree $_{27}$ is the name used by the owner to denote the tree and $I$ is the name used by the owner to designate himself (and, of course, himself is different for each agent).

- in the same way, one can express "Paul counts as a license holder" by $i$ :LicenseHolder(Paul). 
We see in the last example that the notion of role in the organizational sense, being for an agent or an object, is naturally expressed using contextual categorizations.

The introduction of the places as particular objects allows naturally to introduce the roles of space areas. Thus an expression as $i: P r o t e c t e d Z o n e\left(\operatorname{area}_{7}\right)$ allows to categorize the place area $_{7}$ as a protected zone from the point of view of $i$. In geography, it is commonly admitted that a territory is defined as a socially appropriated area. Intuitively, we propose to account for this definition by saying that an ontology $O$ is the expression of a socially or individually constituted point of view, and then that the set of places categorized by the concepts of $O$ constitutes his territory. The following definition formulates this intuitive description.

Definition 2 The set of places $c_{i}: l_{j}$ mentioned in the assertions of the form $\langle$ concept $\rangle\left(c_{i}: l_{j}\right)$ of the ontology $O_{c_{1}}$ is called the territory of $c_{1}$.

The figure 1 illustrates some territories in our application. The park administration, customary lineage and VOI correspond to institution territories. In this case, the park administration and the lineage do not need to decompose the area into subareas. For the lineage, it could be a sacred, forbidden zone. The VOI defines protected zones, cropping zones, etc.. Notice the introduction of territories from the point of view of agents as well. Hence, the villagers only consider the roads between the villages. The ecologist is not an agent within the model, although he defines the notion of habitat to account for flora and fauna dynamics.

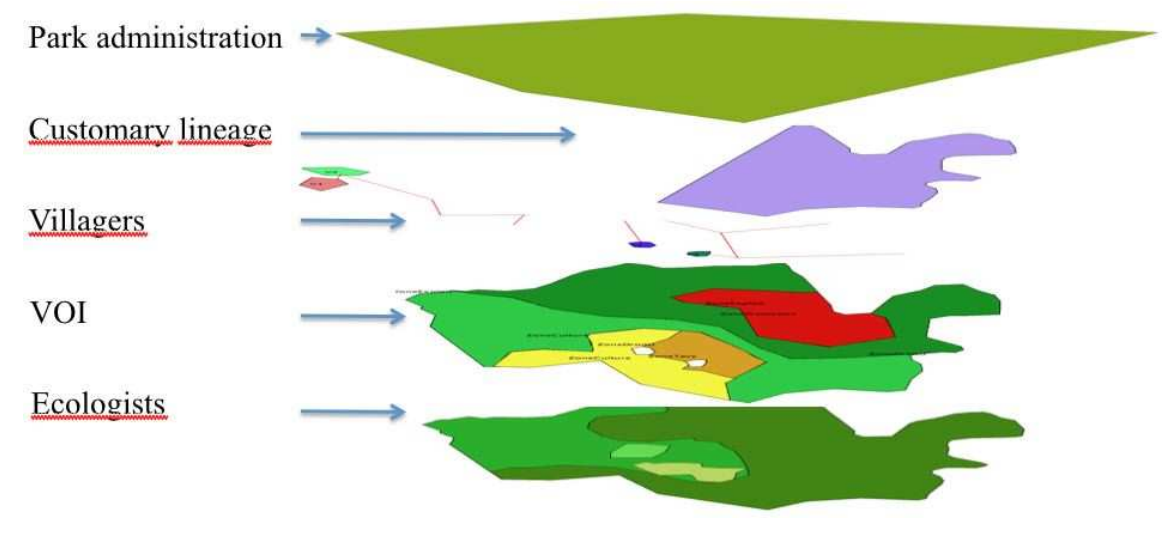

Figure 1: The various territories.

Finally, we define an ontology as a triple $O_{i}=\left\langle\mathbb{L}_{i}, \mathbb{T}_{i}, \mathbb{C}_{i}\right\rangle$ where $\mathbb{L}_{i}$ is its language, $\mathbb{T}_{i}$ is the set of terminological axioms and $\mathbb{A}_{i}$ is the set of its assertions.

The semantics of a family of ontologies $O_{i}$ is defined by a family $M$ of local interpretations ${ }^{1} \quad{ }_{i}=$ $\left\langle\mathscr{A}_{i}, \mathscr{R}_{i}, \mathscr{P}_{i}, \mathscr{L}_{i}, \quad\right.$ i $\rangle$ where:

- $\mathscr{A}_{i}$ is a set of agents;

- $\mathscr{R}_{i}$ is a set of objects;

- $\mathscr{P}_{i}$ is a set of activities;

- $\quad \mathscr{L}_{i}$ is a set of places endowed with a topology;

- $\quad i$ is the semantical function defined as follows:

- ${ }_{i}\left(c \in\right.$ ARole $\left._{i}\right) \subseteq \mathscr{A}_{i}$

- ${ }_{i}\left(c \in\right.$ RRole $\left._{i}\right) \subseteq \mathscr{R}_{i}$

- ${ }_{i}\left(c \in A c t_{i}\right) \subseteq \mathscr{P}_{i}$

- ${ }_{i}\left(c \in \operatorname{Loc}_{i}\right) \subseteq \mathscr{L}_{i}$

- ${ }_{i}\left(r \in \mathbb{P}_{i}\right) \subseteq \mathbb{O}_{i} \times \mathbb{O}_{i}$

- ${ }_{i}\left(o \in A_{i}\right) \in \mathscr{A}_{i}$

\footnotetext{
${ }^{1}$ It is mainly this locality that grounds the contextual feature of these ontologies.
} 


$$
\begin{array}{ll}
\text { - } & i\left(o \in R_{i}\right) \in \mathscr{R}_{i} \\
\text { - } & i\left(o \in P_{i}\right) \in \mathscr{P}_{i} \\
\text { - } & i\left(o \in L_{i}\right) \in \mathscr{L}_{i} \\
\text { - } & i\left(i \in \mathbb{I}_{i}\right) \in M \\
\text { - } & i(\neg c)=\left\{x \in \mathbb{O}_{i} \mid \neg(x \in i(c))\right\} \\
\text { - } & i\left(c_{1} \sqcup c_{2}\right)=\left\{x \in \mathbb{O}_{i} \mid x \in \quad i\left(c_{1}\right) \vee x \in \quad\left(c_{2}\right)\right\} \\
\text { - } & i\left(c_{1} \sqcap c_{2}\right)=\left\{x \in \mathbb{O}_{i} \mid x \in i\left(c_{1}\right) \wedge x \in i\left(c_{2}\right)\right\} \\
\text { - } & i(\exists r . c)=\left\{x \in \mathbb{O}_{i} \mid \exists y,\langle x, y\rangle \in \quad i(r)\right\} \\
\text { - } & i(\forall r . c)=\left\{x \in \mathbb{O}_{i} \mid \forall y,\langle x, y\rangle \in i(r)\right\} \\
\text { - } & i(i: c)=j(c) \cap \mathscr{O}_{i} \text { where } i(i)=j
\end{array}
$$

The last definition allows defining the semantics of a reference to the expression within another ontology. It depends on the possibility to actually designate that ontology $(i(i) \neq \perp)$ and to share, at least partially, the domain of discourse.

To deal with objects and agents positions, we add to $\mathbb{P}_{i}$ a position relationship between a place and an individual. The semantics of the position relationship is given by: ${ }_{i}($ position $) \subseteq\left(\mathscr{R}_{i} \cup \mathscr{A}_{i}\right) \times \mathscr{L}_{i}$.

Finally, the interpretation ${ }_{i}$ is a model of the ontology $O_{i}$ under the following conditions:

- $\quad i=c_{1} \doteq c_{2}$ if and only if ${ }_{i}\left(c_{1}\right)={ }_{i}\left(c_{2}\right)$;

- $\quad i \models c_{1} \sqsubseteq c_{2}$ if and only if ${ }_{i}\left(c_{1}\right) \subseteq i_{i}\left(c_{2}\right)$;

- $\quad i=c(o)$ if and only if $i(o) \in{ }_{i}(c)$;

- $\quad i=r\left(o_{1}, o_{2}\right)$ if and only if $\left\langle{ }_{i}\left(o_{1}\right),{ }_{i}\left(o_{2}\right)\right\rangle \in{ }_{i}(r)$;

This definition is stated differently than in Grossi (2007) where the semantics of an axiom is given by the set of its possible models. It is easy to see that it is equivalent.

\subsubsection{The Regulative Norms}

A regulative norm is expressed in the language $\mathbb{L}_{i}$ of $O_{i}$ and of the form $\left\langle a r_{i}, \bmod _{,} a c t_{i}\right.$, or $\left._{i}, l_{i}\right\rangle$ where:

- $a r_{i} \in$ ARole $_{i}$ is an agent category (role),

- $\bmod$ is a deontic modality (obligation, permission, prohibition),

- $a c t_{i} \in A c t_{i}$ is an activity category,

- $o r_{i} \in$ RRole $_{i}$ is an object category (role) on which the activity applies,

- $l_{i} \in L o c_{i}$ is a place role,

A regulative norm states that an agent considered as playing a given agent role $\left(r_{i}\right)$ has the obligation, permission or prohibition to realize the activity $a c t_{i}$ on the objects playing a given object role $\left(r_{j}\right)$ in a place having the role $l_{i}$. Remind that having a role is equivalent to be contextually categorized as such. For example, given the concepts of User $\left(U_{\text {ser }} \in\right.$ ARole $_{i}$ ) and of Thing (Thing $\in$ RRole $_{i}$ ), as well as the activity ToUse (ToUse $\in A c t_{i}$ ), one can define the norm $\langle$ User, permission, ToUse, Thing,Territory $\rangle$. It expresses that a user has the permission to use a thing all the time on the territory. The name Territory is used instead of "everywhere" because an institution is assumed to be authoritative only on its associated territory. We will see in what follows how to represent that a particular agent plays the role of User, a particular object plays the role of Thing and that, therefore, the norm applies. To simplify, we do not consider conditional norms nor temporal restrictions even if this last extension is taken into account, at least partially, in our implementation.

The natural order on the deontic modalities (obligation $>$ permission $>$ prohibition), as well as the subsumption relation $\sqsubseteq$ induces an order on the norms as given by the following definition:

Definition $3\left\langle r_{i}, \bmod , a c t_{i}, r_{j}, l\right\rangle \leq\left\langle r_{i}^{\prime}, \bmod ^{\prime}, a c t_{i}^{\prime}, r_{j}^{\prime}, l^{\prime}\right\rangle$ if and only if $r_{i} \sqsubseteq r_{i}^{\prime}, \bmod <\bmod ^{\prime}, a c t_{i} \sqsubseteq a c t_{i}^{\prime},, r_{j} \sqsubseteq$ $r_{j}^{\prime}$ and $l \sqsubseteq l^{\prime}$. 


\section{Müller, and Aubert}

Given that $\sqsubseteq$ is a partial order, $\leq$ also is a partial order. This definition is very important to compute the rights to do something somewhere. Intuitively, if we take a set of norms, all the minimal elements of this partial order define the norms that are actually applicable on the activities of the agent. However, they can contradict each other.

\section{AGENTS}

Each agent $a \in A_{S M A}$ is defined in the following way:

Definition 4 The specification of an agent $a$ is a pair $D A_{a}=\left\langle O_{a}, G_{a}\right\rangle$ where:

- $O_{a}$ is an ontology specifying the beliefs of the agent;

- $G_{a}$ is a set of goals expressed in the language $\mathbb{L}_{a}$ of $O_{a}$, as a list of assertions to make true.

This very general definition of goal is enough to express the needs (e.g. access $(I$, $\langle$ Rice, $100 \mathrm{~kg}\rangle))$ as well as the physical (e.g. position $\left(\right.$ house,$\left.l_{34}\right)$ ) or institutional (e.g. ProtectedZone $\left(l_{56}\right)$ ) goals.

The institutions $\mathbb{I}_{a} \in 0_{a}$ are those known to the agent $a$. The affiliation is expressed by an agent counting as playing a given role in the institution. At least, he is member, a role that subsumes all the other roles $r(\forall r, r \sqsubseteq$ Membre). Thus an agent is member of an institution $i$ is expressed by $i: \operatorname{Member}(I)$ (formally, $I$ is in the category Member from the perspective of the institution i). Grossi (2007) is obliged to add a particular predicate rea $(a, r)$ to express that an agent $a$ plays a role $r$. In our formalism, the assertions of the ontology is sufficient. Moreover, this assertion can only be in the institution (only the institution knows that the agent is member), or only in the agent (the agent believes that it has a role in the institution), or in both.

The set of institutions $M$ whose the agent is member, and the territories in which the agent is situated, specify the set of applicable norms in terms of obligation, permission or prohibition to realize a given activity on a given object category. To account for it, we have to define formally the conditions under which a norm $\left\langle a r_{i}, \bmod , a c t_{i}, o r_{i}, l_{i}, q_{i}\right\rangle$ of an institution $i$ is applicable. There are two possibilities:

- the norm is applicable because the agent plays a role in the associated institution;

- the norm is applicable because the agent is situated on a territory regulated by an institution.

The following definitions account for these two cases from the point of view of the agent and from the point of view of the institution.

Definition 5 A norm $\left\langle a r_{i}, \bmod , a c t_{i}, o r_{i}, l_{i}\right\rangle$ of an institution $i$ is applicable from the point of view of the agent $a$ if and only if:

- $\quad i \in \mathbb{I}_{a}$ therefore $a$ knows the institution $i$;

- we can deduce from the axioms of $O_{a}$ that:

- $a r_{j}(I)$ and $a r_{j} \sqsubseteq i: a r_{i}$;

- $a$ knows at least one activity $a c t_{j} \sqsubseteq i: a c t_{i}$;

- $a$ knows at least a category of resource $o r_{j} \sqsubseteq i$ :or ${ }_{i}$;

- $\operatorname{position}(I, l)$ and $l \sqsubseteq i: l_{i}$.

We here assume that $a$ knows something if it exists a name in its language $\mathbb{L}_{a}$ to designate it.

Definition 6 A norm $\left\langle a r_{i}, \bmod , a c t_{i}, o r_{i}, l_{i}\right\rangle$ of an institution $i$ is applicable for an agent $a$ from the point of view of the institution $i$ if and only if:

- $\quad a \in A_{i}$ therefore $i$ knows the agent $a$;

- one can deduce from the axioms of $O_{i}$ that:

- $\operatorname{ar}_{i}(a)$ 
- $a$ knows at least one activity $a c t_{j} \sqsubseteq i: a c t_{i}$;

- $a$ knows at least a category of resource or $_{j} \sqsubseteq i$ :or $_{i}$;

$-\operatorname{position}(a, l)$ and $l \sqsubseteq l_{i}$.

Being applicable from the point of view of an agent, respectively from an institution, does not mean that it will be actually applied. Indeed, an agent may not honor it and an institution, as an agent, may not control it nor apply any sanction for it.

We will now describe in more detail the behavior of the households, respectively the VOI in MIRANA in order to illustrate the use of the proposed formalism.

\subsection{The Households}

The households are characterized by an available workforce and a set of annual needs $\left(\subset G_{\text {household }}\right)$. These needs include quantities of feed, finance, firewood (for cooking and heating), construction wood, medicinal plants and so on. Each year, each household plans its activities and executes them (Figure 2).

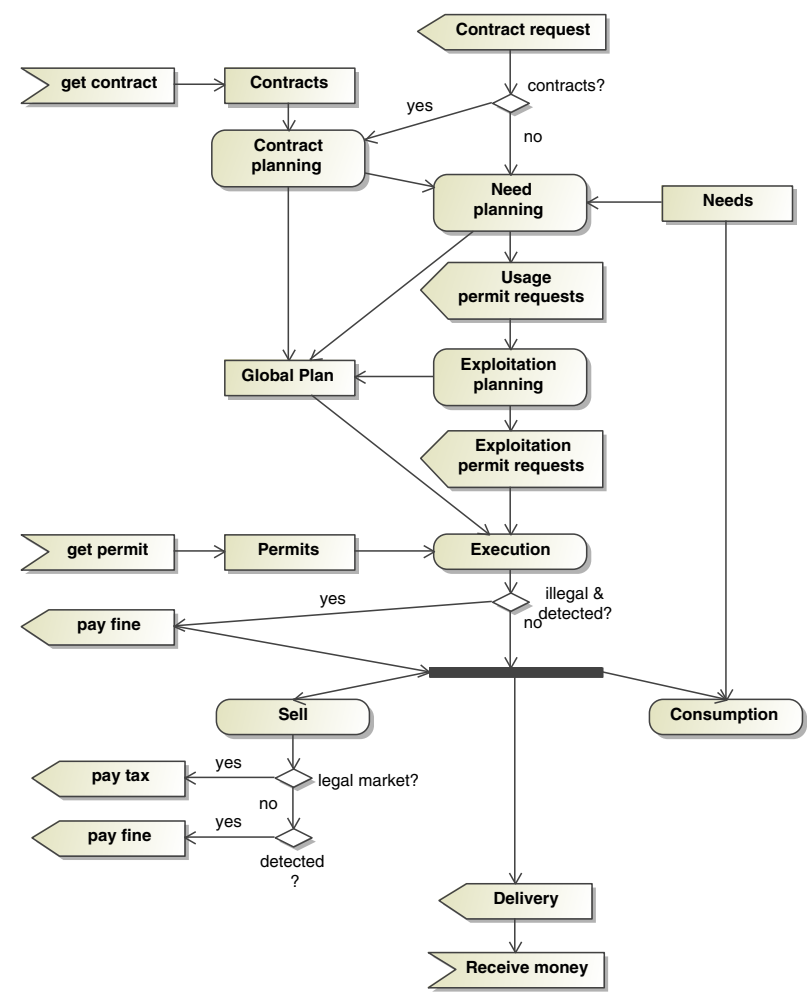

Figure 2: The household behavior activity diagram.

An household starts its cycle by selling all or part of his workforce by asking contracts ("contract request" in figure 2) to the VOI. The planning is thereafter composed of three phases:

1. If the contract request is accepted ("get request"), he receives one or more contracts ("contracts") for lumber jacking, planting or surveillance in order to detect possible norm violations. He has consequently to plan the related activities and to evaluate the remaining workforce. The objective is to sell his workforce to possibly financially cover its needs; 


\section{Müller, and Aubert}

2. Then, he plans his needs up to its available workforce. The use permits regulate the satisfaction of the needs. Therefore he asks for such permits up to the necessary quantities of resources. The objective is to fulfill his needs;

3. Then, if some workforce remains, he plans the production of goods to sell on the market. Here also, the exploitation permits regulate the production and, consequently, are requested for. Here, the objective is to maximize his income.

The three phases produce sequences of actions to perform. These actions are added to a global household's plan ("global plan"). Notice that the behavior of the households does not reduce only to income optimization because we take into account two additional important dimensions of human behavior: 1) The possibility of selling one's workforce although some optimization could be performed on the choice of contract; 2) The auto-consumption that is not based on optimization but on satisfaction only.

After this planning phase, the planned actions will be executed and the results will be delivered to the employer, consumed or sold depending on whether they were produced for the contracts, for satisfying the needs or for selling. The employee gets paid on delivery and the production sold on the legal market is submitted to a tax. At the end of year, every resource that has not be delivered to the employer or consumed is converted to money by being legally or illegally sold, and constitutes the annual financial result of the household.

We will now describe the regulation of the households' activities by the institutions. However, beforehand, we will make three remarks:

1. Each contract constitutes itself a small institution with limited duration (1 year in our simulations). Each contract defines the role of employer and employee with the associated norms in terms of delivery of goods or services, and payment. In our case, the contracts are made with the VOI who institutes the role of license holder for lumber jacking and the role of police for surveillance only to its members;

2. A part of the regulation is externally achieved by a control mechanism. The households in charge of surveillance dedicate a part of their time to monitor the actions of others. If a violation is observed, a fine is applied and the resulting resources are confiscated and given to the VOI.

3. Each household in its decision mechanism internally achieves the other part of the regulation. The result depends on whether the household is legalist or not and will be described hereafter.

At the planning level, each activity has to take place in a certain place $\left(\in L_{\text {household }}\right)$. Therefore, part of the planning phase consists in choosing a place to carry out the activity. The place to be chosen depends on whether the household is legalist or not. If the household is legalist, the activity can only take place on a place where it is authorized from the points of view of all the defined institutions. This authorization depends on the norms applicable to the corresponding territories or zones that overlay upon it. If the household is not legalist, he may consider doing it on places that are not allowed from the point of view of one or more institutions. Notice that the norms can be equally be seen as constraints or resources for action.

At the execution level, the execution of the planned actions to satisfy the needs depends on the use permit from the VOI. If the permit is not granted and the household is legalist, the corresponding action will not be executed, otherwise it will be illegally performed. In the same way, the execution of planned actions for commercial production depends on the exploitation permit from the VOI and follows the same rule. If the action is illegal and the violation is detected, a fine has to be paid and the corresponding resources are confiscated.

This behavior allows checking the impact of the imposed regulations on the financial results (economic sustainability) and the households' satisfaction (social sustainability). If all the households are strictly legalists, the level of satisfaction of the annual needs will be a good indicator of the sustainability of the regulations. If none of the households is legalist, the number of violations (detected or not) will also 


\section{Müller, and Aubert}

constitute a good indicator for the pressure imposed by the regulations. Another indicator could be the relative importance of the goods sold on the formal or informal market.

\subsection{The VOI}

The VOI has the objective, through its associated institution to guarantee a sustainable use of the renewable resources on its territory. As a stakeholder and moral person, the VOI is in charge of implementing the norms of the institution. This implementation of the norms relies on a number of tools:

- The granting of lumber jacking contracts and exploitation licenses to implement the exploitation quotas (the quota is assumed to be defined on the basis of the resources renewal speed);

- The granting of use licenses to implement the use quotas;

- The grants for plantation to compensate the forestry resource losses, and consequently to restore the ecosystem;

- The grants for intensification of the cultivation to increase the crop productivity and possibly reduce the footprint on the ecosystem;

- The granting of surveillance contracts to implement the norm compliance by the households.

Finally, the VOI ensures his own financial sustainability by gathering the fines and taxes, as well as by selling the contracted production and the confiscated goods on the market.

This behavior is summarized in the figure 3 where no sequential order is given to the activities because most of them are triggered by the arrival of the requests, or the order is not important.

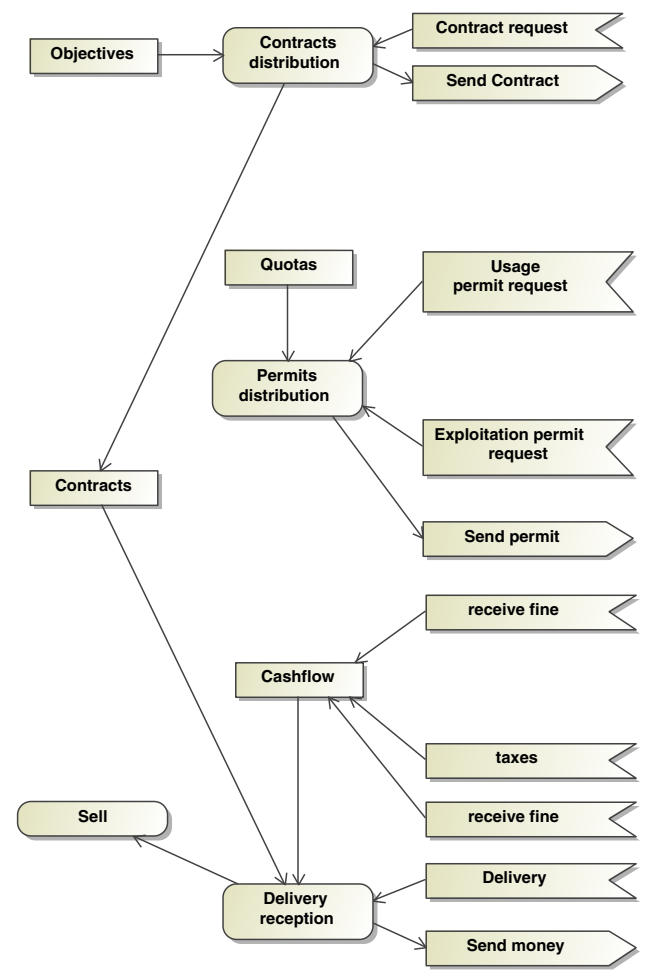

Figure 3: The VOI behavior activity diagram.

At this level, it is possible to parameterize the regulation policies by the institution norms, including the quotas and the implementation policy and to assess the feasibility of the management plan. Therefore, we are globally able to assess the impact of the management plan on the ecological sustainability by 


\section{Müller, and Aubert}

indicators on the ecosystem itself, the economic sustainability of the households and of the VOI, and the social sustainability of the households.

Regarding the VOI economic sustainability, the costs include the surveillance and lumber jacking salaries, and the plantation and intensification incentives, while the revenues include the taxes (both for the permits and on the market sales), the fines, and the timber (both from production and confiscation) sales on the market.

\subsection{Some Results}

The figure 4 illustrates some simulation results over twelve years (120 months) with non-legalist households and only a small fraction of the area with full conservation. The figure 4 a) shows the VOI financial results. The red line represents the tax incomes that are relatively constant over time, producing an increasing net income (green line). The initial negative result is due to the payment of the first salaries. The figure 4 b) shows the evolution of the habitats in percentage of the total surface. There is only a slow erosion of the primary forest. If the degraded land increases, there is similar growth of the secondary forest. The simulation on 60 years (tree growth cycle duration) shows some recovery of the primary forest. However, the figure $4 \mathrm{c}$ ) shows that if we look at the tree species, some are more exploited than others.

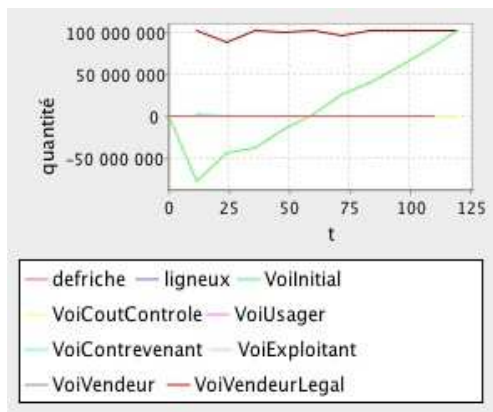

(a) VOI financial results

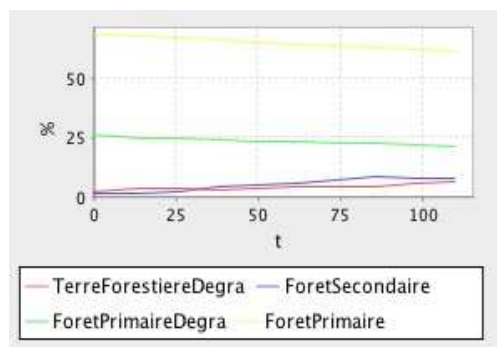

(b) Habitat evolution

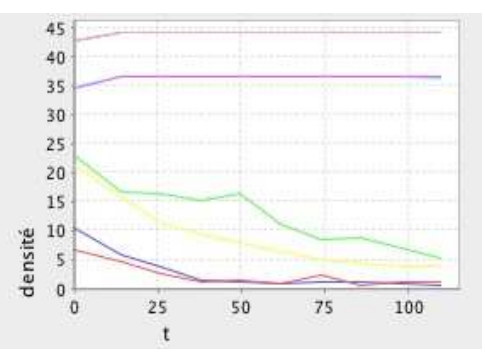

(c) Tree species evolution

Figure 4: Some results.

\section{CONCLUSION}

We have proposed the use of institutions and contextual ontologies to unify the multiplicity of legal interpretations. We have shown that the assertive part of the ontologies account for the notion of role in organizational multi-agent systems. It also allows formalizing the notion of territory as socially appropriated space. Then, we have proposed a formalization of institutions by a set of constitutive norms using the contextual ontologies and of regulatory norms expressed using the corresponding ontology. We also have proposed a formalization of agents as a contextual ontology and a set of goals. The use of contextual ontologies for both the constitutive norms and the agents's perspectives may seem curious. It can be argued in two complementary ways: 1) in our formalization, we do not make the difference between an institution and an organization. Usually, an institution is abstract, while an organization is a concrete group of people implementing an institution. In reality, the distinction is not evident. In our case, each VOI creates his own and unique institution (through its management plan). Therefore, an institution could be considered as a single entity. 2) conversely, an agent identity could be considered as institutionally defined. An ecologist is such as member of a scientific community with its own norms on how to produce science. Forest households share a common social identity in terms of practice or of lineage. Therefore a typology of actors could be represented as a set of institutions as well. The question of representing multiply defined identities, and the resulting hierarchy of organizations and institutions remain to be further pursued.

The dynamics of the agents has been globally defined but a generic specification of the account of norms at the agent level remains to be described. We have, among others, dissociated the institution as a 
structure from the agent implementing the collective objectives through control strategies of norms. The non-regulatory management methods (incentives, taxes, etc.) remain to be formally specified.

\section{REFERENCES}

Aubert, S., J.-P. Müller, and J. Ralihalizara. 2010, July. "MIRANA: a socio-ecological model for assessing sustainability of community-based regulations". In International Congress on Environmental Modelling and Software Modelling for Environment's Sake, edited by D. A. Swayne, 1-8. Ottawa, Canada.

Boella, G., and L. van der Torre. 2004. "Regulative and Constitutive Norms in Normative Multiagent Systems". In Proceedings of Knowledge Representation'04, edited by D. Dubois, C. Welty, and M.-A. Williams, 255-266: AAAI.

Dignum, M. V. 2004, January. A model for organizational interaction: based on agents, founded in logic. Ph. D. thesis, Utrecht University.

Esteva, M., B. Rosell, J. A. Rodriguez-Aguilar, and J. L. Arcos. 2004, July. AMELI: An Agent-Based Middleware for Electronic Institutions, Volume 1, Volume 1. IEEE Computer Society.

Ferber, J., and O. Gutknecht. 1998. "A meta-model for the analysis and design of organizations in multiagent systems". In International Conference on Multi-Agent Systems, edited by Y. Demazeau, 128-135: IEEE.

Grossi, D. 2007. Designing Invisible Handcuffs. Ph. D. thesis, Dutch Research School of Information and Knowledge Systems.

Grossi, D., J.-J. C. Meyer, and F. Dignum. 2008. "The many faces of counts-as: a formal analysis of constitutive rules". Journal of Applied Logic 6:192-217.

Hübner, J. F., J. S. Sichman, and O. Boissier. 2002. "A Model for the Structural, Functional, and Deontic Specification of Organizations in Multiagent Systems". In SBIA, edited by G. Bittencourt and G. Ramalho, $118-128$.

López y López, F., M. Luck, and M. d'Inverno. 2002. "Constraining autonomy through norms". In Proceedings of the first international joint conference on Autonomous agents and multiagent systems: part 2, edited by C. Castelfranchi, 674-681: ACM.

Müller, J.-P. 2010, July. "A framework for integrated modeling using a knowledge-driven approach". In International Congress on Environmental Modelling and Software, edited by D. A. Swayne, 1-8. Ottawa, Canada.

Müller, J.-P., and S. Aubert. 2011, January. "Une ontologie pour une représentation multi-niveau de et par les systèmes sociaux". In Rochebrune 2011, edited by D. Pumain, 1-11.

Ostrom, E. 2005. "Understanding institutional diversity - Google Books". Princeton University Press, Princeton.

Searle, J. 1995. The construction of Social Reality. New York: The Free Press.

World Commission on Environment and Development 1987. Our common future. Oxford University Press.

\section{AUTHOR BIOGRAPHIES}

JEAN-PIERRE MULLER got its $\mathrm{PhD}$ degree in 1987 in Artificial Intelligence, and its privat docent (HDR in France) in 2002. Professor of Computer Science at the University of Neuchâtel (Switzerland) since 1987, he worked in AI and on multi-agent systems. Senior researcher in CIRAD since 2001, he is now working on modeling and simulation of complex systems, applied to socio-ecosystems.

SIGRID AUBERT got its PhD degree in 1999 in Law, and its privat docent (HDR in France) in 2006. Scientist at CIRAD since 2000, she is now working on anthropology of law and the right of biodiversity. She is currently based in Madagascar. 